\title{
Preface
}

\section{Microsurgery Half a Century After Establishment: Global Perspectives}

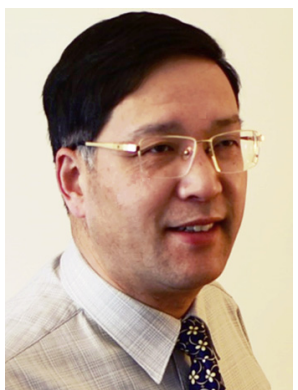

Jin Bo Tang, MD

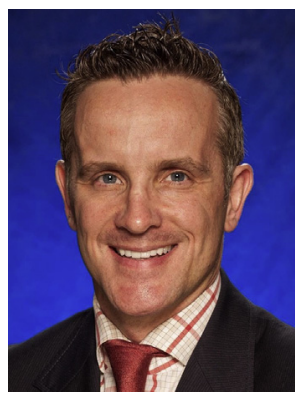

Michel Saint-Cyr, MD, FRCS(C)

Editors

We are proud to assemble and edit this issue offering global perspectives on microsurgery for the jubilee commemoration of the technical establishment and advent of many clinical techniques of microsurgery in the middle of the twentieth century. We are especially grateful to the authors of the review articles presented here; they are world experts in individual topics and have devoted enormous effort to summarizing their innovations and cutting-edge techniques in microsurgery. Of particular note are accounts of work at the very frontier of our field, such as approaches to difficult replantation; transfer of mini-flaps; comprehensive treatment of crush injuries of the hand and forearm; novel vascularized bone grafting; up-to-date techniques for breast, hand, and neck reconstruction; pediatric microsurgery; microlymphatic surgery; and complex reconstruction after tumor excision in the trunk, neck, or extremities.

For this Clinics in Plastic Surgery issue on microsurgery half a century after its technical establishment, we include an editorial on the development of fundamental techniques currently in routine use and about the early days of microsurgery. At the end of the issue, we included two articles on future perspectives, about evolving work on use of robots in microsurgical repair and investigations into composite tissue allografts. We hope to offer readers some perspective on the dramatic changes in microsurgery since the discipline was established, summarize the current state of the field, and provide a glimpse of what may be expected and what challenges remain to be conquered.

Microsurgery has deep roots in plastic surgery, where several of our core techniques originated. Because technical innovations vary widely, with some being very advanced, and new procedures emerging constantly, we feel obliged to remind readers that microsurgery is most powerful when used out of clinical necessity. There are many instances in which simpler and more traditional options achieve similar outcomes, and surgeons should always consider the least complex and technically less demanding options when deciding on treatment. The contents of this issue reflect cutting-edge techniques in microsurgery, which you can use for your own patients when necessary or may inspire you to advance the field in years to come.

We thank all the authors for expert contributions and the publisher for the inspiration to dedicate this issue to commemorating the first half-century of thriving and widespread clinical use of microsurgery. We acknowledge editorial contribution from Drs Daniel Kwan, Jonathan Bass, and Bella Avanessian for their insightful 
comments and review for some of the articles in this issue. Special thanks are given to $\mathrm{Dr}$ Luis Landin for coordinating the article on unique techniques and clinical approaches, and Drs Marco Innocenti and Rohit Arora for thoroughly reviewing their cases and techniques for an article on vascularized bone grafting in this issue. Planning this issue and collecting the articles began in the summer of 2015 and ended in early 2017. Considerable editorial effort was devoted to each of those articles by authors from multiple countries or clinical units, with which we aim to offer comprehensive international views. This effort has been warmly supported by experts from different countries in Europe, America, and Asia. They kindly contributed their wisdom, case materials, and technical points to comprehensive reviews on individual subjects. We humbly acknowledge that the experience of many eminent microsurgeons has not been included, simply because their work was either reflected in recent issues of Clinics in Plastic Surgery or has been well known to our readers, and the work being presented here is fresher.

Development of microsurgery started in the first half of the twentieth century; its foundation for clinical techniques was established about half a century ago. In the mid-1960s and later years, digital replantation and free vascularized tissue transfers came into clinical use; today we enjoy the fruits of the early work. It was an honor to edit this issue. We dedicate it to the many pioneers who established the foundation of the special surgical field we now call microsurgery.

Jin Bo Tang, MD

Department of Hand Surgery Affiliated Hospital of Nantong University The Hand Surgery Research Center Nantong University 20 West Temple Road Nantong 226001, Jiangsu, China

Michel Saint-Cyr, MD, FRCS(C)

Division of Plastic Surgery

Baylor Scott \& White Health

Scott \& White Memorial Hospital MS-01-E443, 2401 South 31st Street

Temple, TX 76508, USA

E-mail addresses:

jinbotang@yahoo.com (J.B. Tang) michel.saintcyr@bswhealth.org (M. Saint-Cyr) 